\title{
A RELAÇÃO DOS PADRÕES ESTÉTICOS NA AVALIAÇÃO PSICOLÓGICA PARA CIRURGIA BARIÁTRICA
}

\author{
Felipe Muniz da Silva ${ }^{1}$
}

RESUMO: As relações contemporâneas limitam o corpo a partir das construções estéticas presentes nas narrativas sociais, e todo corpo que se desvirtua dessa lógica opressora, como o corpo obeso, é condenado a estigmatização por diferentes vias. Atualmente, a cirurgia bariátrica tem sido associada como um meio para a fuga do lugar de estigmatização social a fim de alcançar o corpo magro. Visto isto, o objetivo deste estudo é compreender a importância da investigação dos padrões estéticos na avaliação psicológica para bariátrica, em consonância com a bibliografia levantada. Valendo-se de publicações dos últimos dez anos e em português, foi realizado uma revisão bibliográfica sistemática para coleta de dados, aos quais foram tratados de acordo com os princípios da metassíntese qualitativa. Na literatura analisada foi visto que muitos indivíduos têm a motivação pela realização do procedimento de cirúrgico primordialmente em função da aparência estético, e todos os possíveis ganhos em função desta, perdendo de vista os riscos e as mudanças fisiológicas e psicossociais, e demais complicações que possa vir a ter em busca de um padrão estético. As construções estéticas em torno do corpo obeso precisam ser pensadas no processo de avaliação psicológica pré-bariátrica, posto que o alto risco que a cirurgia oferece deve ser levado em conta no momento de decisão de sua realização. Práxis psicológica, necessita, portanto, corroborar para o maior êxito no pós-cirúrgico, entendo a motivação do indivíduo face esse processo de alto risco.

Palavras-chave: Bariátrica. Estética. Avaliação Psicológica.

Abstract: Contemporary relationships limit the body based on the aesthetic constructions present in social narratives, and any body that deviates from this oppressive logic, such as the obese body, is condemned to stigmatization in different ways. Currently, bariatric surgery has been associated as a means of escaping the place of social stigmatization in order to achieve the thin body. In view of this, the aim of this study is to understand the importance of investigating aesthetic patterns in the psychological assessment for bariatric patients, in line with the literature surveyed. Using publications from the last ten years and in Portuguese, a systematic literature review was carried out to collect data, which were treated according to the principles of qualitative meta-synthesis. In the literature analyzed, it was seen that many individuals are motivated to perform the surgical procedure primarily due to the aesthetic appearance, and all the possible gains due to this, losing sight of the physiological and psychosocial risks and changes, and other complications that may come to have in search of an aesthetic standard. The aesthetic constructions around the obese body need to be considered in the pre-bariatric psychological assessment process, since the high risk that the surgery offers must be taken into account when deciding to perform it. Psychological praxis, therefore, needs to corroborate for the greatest success in the post-surgery period, I understand the individual's motivation in the face of this high-risk process.

Keywords: Bariatric. Aesthetics. Psychological Assessment.

\footnotetext{
I Psicólogo, especialista em psicologia social, psicologia clínica e avaliação psicológica, mestrando pelo programa de pós-graduação em Letras: linguagens e representações da Universidade Estadual de Santa Cruz. E-mail:psi.felipemuniz@gmail.com.
} 


\section{INTRODUÇÃO}

As significações e ritos propostos pelos ordenamentos sociais, tornou o corpo como a intermediação de suas codificações em relação a aparência estética, trazendo uma coletânea de representações que delineiam e determinam os sentidos de beleza, saúde, sedução (DE VILHENA; MEDEIROS; DE VILHENA, 2005).

Tendo em vista a legitimação de um padrão estético, o corpo considerado enquanto belo é exposto pelos mais diversos meios comunicacionais e associado a valores positivos que traduzem discursos de vinculação social, isto é, o pertencer desse corpo no seio social só será possível se caso a felicidade individual, entendida como o alinhamento aos padrões estéticos, for alcançada pelos sujeitos (DE VILHENA; MEDEIROS; DE VILHENA, 2005).

As atitudes que personificam a relação com feiura, ou seja, o que a sociedade limita e desconsidera como belo, produz vínculos sociais esfacelados ou até rompidos em razão da não adequação da sua aparência corporal (VILHENA; MEDEIROS; DE VILHENA, 2005).

Com uma visão reducionista e patologizante, os padrões estéticos atuais remontam para uma estética que sentencia a obesidade em seu âmbito biológico e comportamental (PAIM; KOVALESKI, 2020). Falta de autocuidado, negligência em relação a si mesmo são exemplos da teia constitucional discursiva que explicitam o corpo obeso como motivo para remorso, vergonha e culpa, posto que é um corpo demarcado pela ridicularização de suas dimensões e inadequação diante da práxis social (PAIM; KOVALESKI, 2020).

O estigma que se cria em função da desvalorização do corpo obeso para a sociedade pode ser traduzido por julgamentos que pressupõe que a pessoa obesa é adoecida e infeliz, pois a sensação de ser um fracasso, estar descuidada e ser inapta para a realização de atividades, são incorporados, portanto, naturalizados pelo imaginário social em relação a pessoa obesa (PAIM; KOVALESKI, 2020).

O corpo magro torna-se saudável, enquanto o corpo obeso passa a ser um corpo doente, que necessita de intervenções para se tornar saudável (PAIM; KOVALESKI, 2020). Sendo assim, a abordagem em relação ao peso deve ser interposta pelo automonitoramento, dietas restritivas, uso de fármacos e intervenções cirúrgicas, como a cirurgia bariátrica (PAIM; KOVALESKI, 2020). 
Por sua vez, a cirurgia bariátrica ou cirurgia de antiobesidade, pode ser entendida como um procedimento cirúrgico que limita a capacidade gástrica e/ou que pode interferir na digestão, tendo o objetivo principal de controle da obesidade mórbida em longo prazo (PORIES, 2003 apud OLIVEIRA; LINARDI; AZEVEDO, 2004).

Há certas restrições para a realização do procedimento cirúrgico, havendo a necessidade de um processo de avaliação psicológica para atestar condições cognitivas, psíquicas, sociais e emocionais mínimas, tendo em vista que esta cirurgia afeta diferentes esferas vivenciais do sujeito (FLORES, 2014).

A motivação por trás da realização da cirurgia bariátrica, revela a necessidade que o indivíduo coloca na adequação aos padrões estéticos vigentes, tendo como pressuposto o seu conteúdo fantasioso em relação ao seu pós-cirúrgico e tudo aquilo que será capaz de desempenhar (MARCHESINI, 2010).

Partindo do pressuposto do risco que a cirurgia bariátrica oferta para o indivíduo face a sua motivação pelo ganho estético-social, têm-se como pergunta norteadora: qual a relevância da investigação dos padrões estéticos na avaliação psicológica para cirurgia bariátrica, segundo a bibliografia pesquisada?

Compreendendo a temática exposta, o presente estudo justifica-se pela necessidade de embasar discussões sobre os padrões estéticos e sua ligação com o campo prático-teórico da avalição psicológica para cirurgia bariátrica, visto que a simbologia em torno do corpo estético faz-se presente nas narrativas sociais e podem impactar de maneira negativa o funcionamento psíquico de indivíduos pós-bariátricos, havendo, portanto, a necessidade de um olhar mais atento do psicólogo em prol da saúde mental do público.

O objetivo geral deste estudo é compreender a importância da investigação dos padrões estéticos na avaliação psicológica para bariátrica, em consonância com a bibliografia levantada. E os objetivos específicos se fundamentam em identificar o papel da estética como fator de motivação para a realização da cirurgia bariátrica, de acordo com a bibliografia analisada e entender a implicação simbólica da estética na avaliação psicológica para cirurgia bariátrica.

\section{REFERENCIAL TEÓRICO}

Dentre as mais diversas e distintas organizações sociais, o corpo é alocado à um lugar de representações simbólico-estéticas, aos quais são elaborados pelos discursos 
culturais, políticos e científicos vigentes, reverberando no desenvolvimento das relações sociais e nos delineamentos dos modos de subjetividade construídos (DANTAS, 20II).

Dentre as narrativas interpostas no contexto atual, o conceito de beleza se vê homogêneo, uniforme para as massas, incitando e valorizando o consumo de um biotipo, ao mesmo tempo em que rejeita tudo o que foge dessa lógica (RIBAS; CALEIRO, 20I2). Tendo em vista a perspectiva do individualismo moderno, descrito por Ribas e Caleiro (2012), os discursos sociais acusam uma necessidade de controle sobre a construção do sujeito, ao qual se vê pressionado e cede a tentativa de se encaixar na concepção social de beleza, consumindo o que tem disponível para aproximar-se dela.

Este prisma de pensamento fomenta as mudanças capitalistas e a disseminação de normas e imagens do século XX, aos quais propuseram a fabricação de uma beleza rentável ao mercado de produtos e procedimentos para modelagem do corpo (FIGUEIREDO; NASCIMENTO; RODRIGUES, 2017).

Com um discurso binário, este contraste estético proposto pelo gênero remonta a uma doutrinação sobre como as práticas subjetivas do corpo devem ser apresentadas para a sociedade, ilustrando a necessidade de o público feminino mostrar-se mais frágil e com medidas mais reduzidas, enquanto o público masculino deve se apresentar de maneira forte, voluptuosa e musculosa, por exemplo (BARBOSA; DA SILVA, 2016).

Tal fato pode ser atestado em conteúdos imagéticos, como os anúncios, em que possui um foco em um corpo feminino magro e considerado bem-vestido, de pele e cabelos impecáveis, com vistas a passar uma imagem de sucesso profissional, status pessoal e casamento harmonioso (RIBAS; CALEIRO, 20I2). Enquanto o corpo masculino traduz ser bem-sucedido pelo uso de roupas alinhadas e corpo malhado (RIBAS; CALEIRO, 20I2). Tem-se, portanto, a exibição insistente de mulheres magras e homens de porte físico robusto (RIBAS; CALEIRO, 20I2).

E pensando em pertencer aos ordenamentos sociais, homens e mulheres se veem em uma espécie de maratona em busca da possível descrição de beleza regida na sociedade, estando aptos a comprometer a sua saúde física e/ou mental para alcançar os padrões estéticos a preços altos (RIBAS; CALEIROS, 2012).

Sendo assim, a cobrança pela aparência com medidas reduzidas é requisitada cotidianamente aos sujeitos devido a ênfase reiterada pela ótica da sociedade, em que acaba 
por construir estigmas e valores em torno do que compreende como inaceitável, a exemplo do corpo obeso (MACEDO et al., 2015).

A supervalorização do corpo esguio converte a gordura enquanto uma falência moral, tornando a pessoa obesa estigmatizada por apresentar um corpo socialmente inadequado e indesejável (MATTOS; LUZ, 2009).

Essa desqualificação do sujeito obeso face a sua aparência, lhe atribui uma série características estigmatizantes que lhe coloca à margem social, sendo considerado como diferente, depreciado, estranho e desvalorizado em relação ao demais (MATTOS; LUZ, 2009). Portanto, a discriminação e menor aceitação social acabam por estigmatizar a figura obesa quando compreende que o seu corpo é um constrangimento social (MATTOS; LUZ, 2009)

Para Macedo et. al. (2015), “os indivíduos com obesidade precisam adaptar-se a um mundo que possui valores, padrões, regras e estruturas em que o excesso de peso e as comorbidades provavelmente são fatores limitantes e estigmatizantes" (p. 506). O excesso de peso leva a inadequação em consonância com os padrões de beleza vigentes, causando ao indivíduo obeso um maior sofrimento psíquico e provocando uma esfera existencial de invisibilidade na práxis sociais, visto que pessoas com obesidade cursam menor número de anos escolares e possuem menor chance de desenvolver um relacionamento estável, além de menor probabilidade de serem aceito em vagas de emprego, segundo Macedo et. al. (2015).

Diante da exacerbada cobrança social em relação a magreza, o indivíduo obeso vê sua percepção corpórea alinhada com os ditames da sociedade, tendo, portanto, o seu autoconceito atravessado pela depreciação e distorção de significado estético, ou seja, uma preocupação e uma insatisfação quando vê que o seu corpo não é o mesmo valorizado pela sociedade (MACEDO et. al., 2015).

O que é evidenciado, é uma percepção corpórea decorrente da interação entre os elementos pertencentes a uma rede de valores que privilegia certas medidas corporais, e em função disso tem muitas vezes a visão dimensional de si depreciada pelas repercussões psicossociais, isto é, um reflexo que causa sofrimento, sendo este ilustrado pela "vivência de sentimentos como tristeza, vergonha, inibição e frustração provocados pelo corpo obeso determina que para se mantenha bonito e aceito é preciso ser magro, condição que garante o bem estar consigo e com os outros" (MACEDO et. al., 2015, p. 508). 

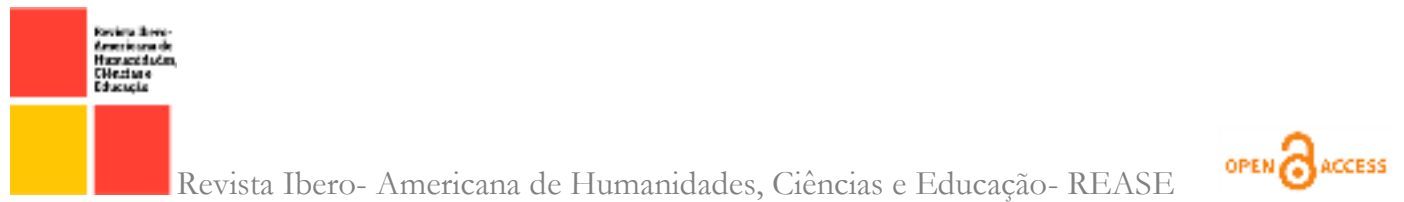

Em estudo, a desfiguração da autoimagem atestou a relação de discriminação de pessoas obesas versus a idolatria da magreza, ao mesmo tempo em que gera uma insatisfação pela sua aparência (MACEDO et. al., 2015). O que é visualizado, é uma cobrança exacerbada em relação a magreza dentro de um ideal estético que segrega e que coloca a gordura corporal como um agente negativo (ARAÚJO et al., 2014).

A busca pelo corpo mais magro e torneado em função da inadequação e insatisfação em relação a própria imagem, têm fomentado a busca por procedimentos que se alinham a proposta social, e a cirurgia bariátrica pode ser um caminho possível para um "renascimento" estético, de acordo com o significado de beleza atual.

A cirurgia bariátrica é atualmente o tratamento mais efetivo para a obesidade grau III, e a sua finalidade é aumentar a qualidade de vida da pessoa obesa, seu tempo de vida e consequentemente dirimir questões de ordem psicossocial, já que o excesso de peso é uma condição clínica complexa com consequência psicológicas e sociais (COSTA et. al., 2008).

Para Oliveira, Linardi e Azevedo (2004), pessoas obesas apresentam maiores níveis de sintomas depressivo, alimentares, ansioso e de transtorno da personalidade, visto que apresentam um sofrimento psicológico decorrente do estigma social sobre o seu peso, o que pode ser um fator motivador para a procura da cirurgia antiobesidade.

A cirurgia bariátrica é um procedimento complexo, irreversível e com alto risco de complicação, necessitando que o paciente conheça todas as suas minúcias do pré ao póscirúrgico devido ao seu alto impacto nas searas biológicas, psicológicas e sociais do paciente, fazendo-se necessário o acompanhamento psicológico em todas as fases para a possibilidade do menor agravo possível na saúde mental (OLIVEIRA; LINARDI; AZEVEDO, 2004).

As rápidas mudanças ocasionadas pela bariátrica acabam por demandar um grande preparo emocional e uma significativa habilidade resiliência, posto que as transformações se estendem para os hábitos alimentares, medidas do próprio corpo e a criação de uma nova identidade em função da nova aparência corporal e todas as suas implicações psicológicas e sociais, havendo, portanto, a necessidade do trabalho de um profissional da psicologia nesse processo (OLIVEIRA; LINARDI; AZEVEDO, 2004).

A práxis da psicologia na equipe multidisciplinar pré-operatória deve atestar condições mínimas para a realização da cirurgia bariátrica, se atendo ao uso de substâncias, presença de quadro psicóticos e outras desordens psíquicas que comprometem o 
entendimento do procedimento, como esquizofrenia e quadros demenciais, além de verificar a presença de suporte social, elementos estressores cotidianos, relações de compulsão, história de vida, ideação suicida e expectativas do pós-operatório (FLORES, 2014).

O levantamento de informações da esfera psíquica e emocional, com vistas ao entendimento da indicação ou não para o encaminhamento cirúrgico, deve ser intermediado pela avaliação psicológica, designada a investigação dos aspectos emocionais, cognitivos e psiquiátricos do candidato à bariátrica, sendo a entrevista clínica e a testagem psicológica, dois importantes instrumentos para o entendimento do funcionamento psicológico do indivíduo (FLORES, 2014).

Ao compreender a importância da avaliação psicológica no pré-operatório, Flores (2014) discute que esta aumenta consideravelmente a chance de sucesso no período pósoperatório em razão do entendimento sobre a dinâmica psíquica e da possibilidade da emergência de mudanças comportamentais e paradigmáticas, para além de um momento de psicoeducação sobre as mudanças implicadas ao procedimento de cirúrgico.

Atualmente, não existe um protocolo a fim de nortear o processo de avaliação psicológica para cirurgia bariátrica, fazendo com que haja uma dificuldade em saber quais os construtos implicados no procedimento cirúrgico, bem como as melhores ferramentas a serem utilizadas no processo de levantamento informacional (FLORES, 2014). Sendo assim, a ausência desse norte protocolar influencia diretamente na coleta de informações, podendo deixar de fora a avaliação de variáveis fundamentais que influem até na motivação do sujeito para a realização de um procedimento cirúrgico complexo. Tal motivação, pode ser traduzida a partir da adequação estético-corporal vigente nos ordenamentos sociais.

Para Marchesini (2010), a compreensão da busca do indivíduo pela cirurgia de antiobesidade é primordial no processo, pois "as motivações que levam os indivíduos a procurar a cirurgia bariátrica, são em sua maioria, calcadas nos retornos sociais, nos padrões estéticos e no desejo psicológico de mudança de vida" (p.I13).

A compreensão sobre os fatores motivadores pode auxiliar em todo o processo de avaliação psicológica, inclusive quando houver a necessidade de trabalhar as expectativas do indivíduo em relação ao procedimento cirúrgico, posto que a maioria se relaciona com um conteúdo ora fantasioso em relação a novas possibilidades de vida, mas "uma vez 
concretizada a mudança de vida, a motivação se acaba, mas não garante a satisfação existencial [...]” (MARCHESINI, 2010, p. II3)

A partir da mudança para o novo corpo, isto é, uma nova imagem corporal, o indivíduo passa a sentir-se reconhecido e valorizado dentro do contexto social ao qual pertence, pois revalida a identidade e o sentido existencial do sujeito quando este consegue executar suas funções e ocupar um papel social, isto é, quando o seu corpo deixa de se tornar uma penumbra social, saindo do lugar da estigmatização e invisibilidade, e sua identidade flui pelas atividades que realiza (TAVARES, 2003).

O que se infere é o quanto a sociedade limita o ser humano a partir de um padrão de beleza estabelecido, e muitas vezes impossível de atingir, que impõe uma ordem ilógica de que o corpo precisa ser remodelado, mutilado e transformado se caso desejar sair do lugar da estigmatização.

\section{METODOLOGIA}

O presente estudo é embasado nos procedimentos metodológicos referentes à revisão bibliográfica sistemática, baseada na análise de metassíntese qualitativa, uma vez que a proposta desta pesquisa é entender o que a literatura aborda sobre a relação dos padrões estéticos presentes na avaliação psicológica para cirurgia bariátrica.

A pesquisa bibliográfica é rica em materiais já elaborados e permite que o pesquisador tenha um maior conhecimento sobre os fenômenos a serem analisados, definindo assim uma linha de pesquisa a que se pretende conhecer (GIL, 2002). Esse tipo de procedimento metodológico, segundo Caminha e Gomes (2014), tem caráter criterioso e rigoroso, com base em consistente evidência científica quando possui uma pergunta norteadora clara e objetiva. Quando realizada, permite com que outros pesquisadores utilize os seus resultados e discussões com uma maior confiabilidade científica em determinada área específica (CAMINHA; GOMES, 2014).

Para tanto, o tratamento dos dados embasado na metassíntese qualitativa, "visa produzir interpretações ampliadas de resultados ou achados de estudos qualitativos obtidos por estudos primários [...], os quais são selecionados atendendo a um interesse específico do pesquisador [...]” (FIORENTINI, 20I4, p. 78). A intenção é produzir uma nova explicação e compreensão do tema investigado, o que Fiorentini (2014) chama de metainterpretação. Vale a pena ressaltar que a operacionalização da metassíntese é 
intermediada previamente pela análise metatéorica, referente aos conceitos, teorias e explicações utilizadas pelos autores em suas respectivas publicações me relação ao fenômeno pesquisado, e pelo metametodo, que se delineia pela descrição e considerações das metodologias utilizadas nas respectivas publicações a serem analisadas.

As etapas do processo de revisão da literatura já publicada foram: formulação da pergunta norteadora da pesquisa, delimitação das fontes de dados, eleição das palavraschave para a busca nos bancos de dados, busca e triagem dos resultados de acordo com os critérios de inclusão e exclusão, organização dos resultados, leitura e avaliação individual dos dados obtidos, síntese e interpretação dispostas em tabela da literatura encontrada, e finalmente, a discussão dos resultados.

Haja vista, a coleta de dados foi realizada entre 04 e os do mês de Dezembro no ano de 202I, valendo-se das seguintes bases de dados: Scientific Eletronic Library Online (SCIELO), Biblioteca Virtual em Saúde (BVS) e Portal de Periódico da CAPES. Foi definido como critério de inclusão: artigos científicos e trabalhos de conclusão de pósgraduação Latu Sensu e Stricto Sensu, publicados nos últimos io anos, ou seja, de 2011 a 2021, devido a importância de versar com conhecimentos científicos relativamente recentes. Outro critério a se considerar diz respeito a estudos no campo da Psicologia, que se coadunam com a temática. Foram incluídos estudos que apresentassem relação e discussão entre as seguintes palavras-chave: Padrões Estéticos, Avaliação Psicológica; Cirurgia Bariátrica, que foram publicados em língua portuguesa. Estudos que não satisfazem os critérios de inclusão supracitados, estudos repetidos contidos nas diferentes bases de dados ou não disponibilizados na íntegra, foram excluídos desta pesquisa.

\section{RESULTADOS}

A busca nas bases de dados, segundo os critérios eleitos, identificou inicialmente um total de 7 publicações, sendo todas elas artigos científicos e advindos do portal de periódico da CAPES. As demais fontes de busca resultaram em o publicações encontradas. Foram excluídos dois trabalhos em função da sua indisponibilidade na íntegra, e um trabalho por não abordar a temática em questão, restando, portanto, 4 publicações selecionadas.

As 4 publicações selecionadas foram analisadas na íntegra e verificada a pertinência em relação a temática proposta. A publicação excluída, lida na íntegra, tratava sobre a 
qualidade de alimentação e montante de consumo de carboidratos em praticantes de musculação, não satisfazendo os critérios de inclusão em relação ao tema abordado. $O$ diagrama do processo de identificação e inclusão de publicações na metassíntese é apresentado a seguir:

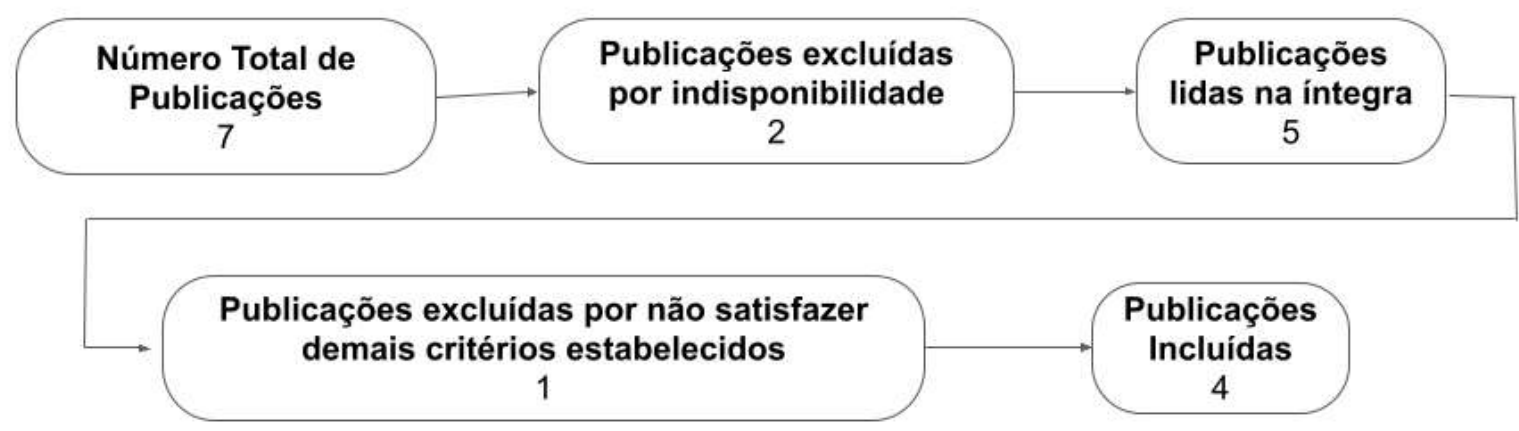

FIGURA 1 - Diagrama do Processo de seleção de publicações para metassintese

\section{ANÁLISE METATEÓRICA}

Os quatro artigos qualitativos selecionados, foram lidos e analisados na íntegra e trazem os seguintes delineamentos e objetivos de acordo com as respectivas amostras: estudo de caso com 6 participantes entre 24 e 47 anos, sendo selecionados por considerar a sua condição pregressa de obesidade que já tentaram vários tipos de tratamento, e com o objetivo de conhecer as repercussões da cirurgia bariátrica na saúde de um grupo de indivíduos a ela submetidos (MARCELINO; PATRICIO, 20II), pesquisa de campo em um grupo de orientação e acompanhamento de pacientes submetidos a cirurgia bariátrica, a fim de analisar alguns conflitos envolvidos nas experiências de pessoas submetidas a cirurgia bariátrica, após suas tentativas de controle do peso (BENTO; MÉLO, 2019), pesquisa exploratória com dez obesos adultos entre 31 e 65 anos em processo de cirurgia e realizado acompanhamento psicológico, pensando em descrever as características do processo reflexivo de obesos candidatos à cirurgia bariátrica, a partir de sua conversa interna (EHRENBRINK; SOUZA, 2018), e por fim, mais um estudo de campo que selecionou ro participantes que foram submetidos à técnica cirúrgica de by-pass gástrico que estivessem apresentado reganho de peso, com o objetivo de analisar alterações na percepção corporal e medo do reganho de peso na amostra pesquisada (MARCHESINI; ANTUNES, 2017). Na tabela abaixo é possível visualizar as publicações selecionadas: 


\begin{tabular}{|c|c|c|c|c|c|}
\hline Título & $\begin{array}{c}\text { Autor(es)/ } \\
\text { Ano }\end{array}$ & $\begin{array}{l}\text { Delinea } \\
\text { mento }\end{array}$ & Coleta & Análise & Objetivo \\
\hline $\begin{array}{l}\text { A complexidade da obesidade e } \\
\text { o processo de viver após a } \\
\text { cirurgia bariátrica: uma questão } \\
\text { de saúde coletiva }\end{array}$ & $\begin{array}{l}\text { MARCELINO; } \\
\text { PATRICIO, } \\
2011\end{array}$ & $\begin{array}{l}\text { Estudo de } \\
\text { caso }\end{array}$ & $\begin{array}{l}\text { Entrevista em } \\
\text { profundidade e } \\
\text { formulário com } \\
\text { questões abertas }\end{array}$ & $\begin{array}{l}\text { Levantamento de } \\
\text { categorias e leitura } \\
\text { reflexiva }\end{array}$ & $\begin{array}{l}\text { Conhecer as repercussões da cirurgia } \\
\text { bariátrica na saúde de um grupo de } \\
\text { individuos a ela submetidos }\end{array}$ \\
\hline $\begin{array}{l}\text { "A cabeça continua de gordo": } \\
\text { dilemas da gestão de si de } \\
\text { pessoas submetidas a cirurgia } \\
\text { bariátrica }\end{array}$ & $\begin{array}{c}\text { BENTO; } \\
\text { MÉLO, } 2019\end{array}$ & $\begin{array}{l}\text { Pesquisa de } \\
\text { campo }\end{array}$ & $\begin{array}{l}\text { Observação de } \\
\text { participante e } \\
\text { entrevistas } \\
\text { semiestruturadas }\end{array}$ & - & $\begin{array}{l}\text { Analisar alguns conflitos envolvidos } \\
\text { nas experiências de pessoas } \\
\text { submetidas a cirurgia bariátrica, após } \\
\text { suas tentativas de controle do peso. }\end{array}$ \\
\hline $\begin{array}{l}\text { Autoconsciência e conversa } \\
\text { interna de adultos obesos à } \\
\text { espera de cirurgia bariátrica: } \\
\text { Um estudo qualitativo }\end{array}$ & $\begin{array}{l}\text { EHRENBRINK; } \\
\text { SOUZA, } 2018\end{array}$ & Exploratório & $\begin{array}{l}\text { Protocolo de } \\
\text { eliciamento de } \\
\text { verbalização da } \\
\text { conversa interna }\end{array}$ & $\begin{array}{l}\text { Fenomenologia- } \\
\text { semiótica }\end{array}$ & $\begin{array}{l}\text { Descrever as características do } \\
\text { processo reflexivo de obesos } \\
\text { candidatos à cirurgia bariátrica, a } \\
\text { partir de sua conversa interna. }\end{array}$ \\
\hline $\begin{array}{l}\text { A percepção do corpo em } \\
\text { pacientes bariátricos e a } \\
\text { experiência do medo do } \\
\text { reganho do peso }\end{array}$ & $\begin{array}{l}\text { MARCHESINI; } \\
\text { ANTUNES, } \\
2017\end{array}$ & $\begin{array}{c}\text { Pesquisa de } \\
\text { campo }\end{array}$ & $\begin{array}{c}\text { Entrevista } \\
\text { semiestruturada }\end{array}$ & $\begin{array}{l}\text { Análise de } \\
\text { Conteúdo }\end{array}$ & $\begin{array}{l}\text { Analisar alterações na percepção } \\
\text { corporal e medo do reganho de peso } \\
\text { em } 10 \text { pacientes submetidos à } \\
\text { cirurgia bariátrica de Fobi-Capella, há } \\
\text { no minimo quatro anos, que tiveram } \\
\text { reganho de pelo menos } 20 \% \text { do peso } \\
\text { perdido }\end{array}$ \\
\hline
\end{tabular}

TABELA 1 - Resultados selecionados

Das publicações, sendo todos artigos científicos, é inicialmente proposto uma discussão em torno da obesidade enquanto uma condição de saúde clínica, apontando que, segundo dados da Organização Mundial de Saúde, a obesidade tem se consolidado cada vez mais nos últimos tempos devido a produção e consumo da alimentação, reverberando na qualidade de vida geral da pessoa obesa e em áreas específicas, como o trabalho e a convivência com o meio social (MARCELINO; PATRICIO, 2oIr; BENTO; MÉLO, 2org; EHRENBRINK; SOUZA, 20I8; MARCHESINI; ANTUNES, 2017).

A obesidade é apontando com maior prevalência no público infantil em um cenário em que cerca de $60 \%$ da população mundial, em um futuro nem tanto longínquo, apresentarão o agravo ou surgimento de algum problema de saúde relacionado à obesidade (MARCELINO; PATRICIO, 20II). Sendo assim, a obesidade possibilitaria a presença de doenças crônicas e demais sofrimentos psicossociais (MARCELINO; PATRICIO, 20II).

Por outro lado, os discursos em torno do corpo obeso não apenas revelam uma problemática configurada apenas nas condições de saúde, já que a apresentação da estética corporal é algo que perpassa as relações sociais e subjetivas que a pessoa obesa mantém, conforme a bibliografia analisada (MARCELINO; PATRICIO, 2oII; BENTO; MÉLO, 2019; EHRENBRINK; SOUZA, 2018; MARCHESINI; ANTUNES, 2017). 
O sucesso intermediado pela relação estética com a magreza corrobora a pressão social em torno de seus atores com vistas ao emagrecimento, gerando sofrimento ao corpo obeso por não ocupar um lugar de representação de beleza em seu contexto e tendo que se enquadrar em uma esfera social que não possibilita acessos para quem foge destes ditames e que, por sua vez, promove e mantém sentimentos de insatisfação em relação a própria imagem, reverberando na pouca autoestima, nos relacionamentos afetivos $e$ no desencadeamento de transtornos alimentares, depressão e ansiedade (EHRENBRINK; SOUZA, 2018; MARCHESINI; ANTUNES, 2017).

Dentro do que é proposto, a obesidade possui um foco no comportamento individual, isto é, um exercício de culpabilização por não atingir o padrão estético, e na era contemporânea, percebe-se um agravamento em função da influência midiática em como a imagem corporal deve se apresentar, uma vez que exibem imagens artificiais, vendidas como reais e possíveis, fazendo com que resulte em um aumento significativo na insatisfação e no sentimento de inadequação da pessoa obesa (BENTO; MÉLO, 2oig; MARCHESINI; ANTUNES, 2017).

Todo esse enlace de práticas sociais em torno da valorização e consideração de um biotipo estético torna à margem quem não se enquadra nessa lógica perversa, e pessoas obesas podem ver se ver estigmatizadas a partir do aparato relacional em diversas ramificações da vida cotidiana, que podem ser traduzidas desde as narrativas culturais até as restrições das vivências rotineiras (EHRENBRINK; SOUZA, 20I8; BENTO; MÉLO, 2019). Sendo assim, o estigma leva as pessoas a procurar alternativas para esconder ou modificar o que o espectro social considera como diferente, com vistas a obter a aceitação nos diversos espaços sociais (EHRENBRINK; SOUZA, 2018).

A ansiedade social perpassada pela pessoa obesa, revela desconforto que sente nas interações sociais, já que a obesidade é tida como uma injustificável falta de autocuidado, isto é, o corpo obeso passa a ser precário de si mesmo, uma ameaça à nossa própria vida, e como consequência, a cirurgia bariátrica tem sido uma opção para fugir do lugar dos estigmas sociais (EHRENBRINK; SOUZA, 2018; BENTO; MÉLO, 2019).

Das opções presentes, a cirurgia bariátrica deve ser considerada como a última alternativa para a redução de peso de pessoas com obesidade mórbida e pessoas com obesidade que apresentam outras complicações em função desta, devido aos riscos biológicos e psicológicos que o indivíduo possa a vir passar, levando em consideração ser 
um procedimento cirúrgico que possibilita uma drástica redução de peso nos primeiros meses pela redução do estômago que se enche rapidamente com poucas quantidades de comida, promovendo alterações na rotina e na forma como o sujeito se percebe (MARCELINO; PATRICIO, 2oII; BENTO; MÉLO, 2019).

A atenção aos riscos cirúrgicos e ao tratamento prolongado devem ser postos nos momentos iniciais de decisão do candidato a cirurgia, já que este indivíduo se relaciona com um conteúdo fantasioso do seu pós-cirúrgico, a exemplo da correlação entre a perda de pessoa e a imagem corporal, em que a diminuição numérica na balança não deve ser entendida como o aumento da autoestima ou a presença da satisfação estética (MARCELINO; PATRICIO, 20I; MARCHESINI; ANTUNES, 2017).

A relevância de um acompanhamento psicológico eficaz nas diferentes etapas do processo se faz necessária em virtude das vivências sociais e psíquicas que o indivíduo manteve ou mantém com o seu próprio corpo, sendo traduzido na aceitação, percepção de si e do mundo e como é no quesito de adaptação a mudança (EHRENBRINK; SOUZA, 2018; MARCHESINI; ANTUNES, 2017).

A partir das perspectivas apontadas nos quatro artigos fica evidente que o corpo obeso se esbarra em suas limitações biológicas, mas não exclui o fato do lugar de estigmatização que ocupa, fazendo com que se visualize a bariátrica como o meio de promover uma maior qualidade de vida em suas diversas égides relacionais, mas para isto, o acompanhamento psicológico no pré e pós-bariátrico se faz fundamental. Não há menção em nenhuma literatura analisada sobre o processo de avaliação psicológica pré-bariátrica, apenas é ressaltado a importância do profissional de psicologia durante todo o processo cirúrgico.

\section{METAMÉTODO}

De acordo com as perspectivas dos respectivos referenciais teóricos utilizados pelos autores, foi visto que os métodos utilizados buscaram explorar uma amostra de pessoas, sendo, portanto, todos submetidos ao comitê de ética para pesquisa com seres humanos.

O método mais frequente utilizado foi a pesquisa de campo, já que esta revela a importância do contato do pesquisador com a realidade ou fenômeno a ser estudado, no manejo que tenta entender como se dá o contexto em sua totalidade (BENTO; MÉLO, 2019). Para o delineamento deste tipo de estudo, a coleta dos dados se por observação de 
participantes e entrevistas semiestruturadas (BENDO; MÉLO, 2019), e no outro estudo somente a entrevista semiestruturada foi utilizada como instrumento para coleta dos dados que foram tratados pela Análise de Conteúdo, método investigativo de pesquisas qualitativas com o objetivo de alcançar a profundidade do sentido das categorias presentes no recorte de realidade estudado (MARCHESINI; ANTUNES, 2017). Desta maneira, a técnica de coleta da entrevista semiestruturada foi a mais utilizada.

O estudo de caso surgiu também como uma possibilidade de delineamento metodológico de pesquisa, se coadunando com a amostra, pois esse tipo de estudo possibilita a compreensão do fenômeno no âmbito da subjetividade de cada sujeito, revelando os aspectos de natureza individual, bem como este se manifesta na coletividade e no universo de significações (MARCELINO; PRATRICIO, 20II). No estudo de caso, foi utilizada novamente a entrevista, mas desta vez, entrevista em profundidade somados a um formulário com questões abertas, direcionados a identificação da história pregressa dos sujeitos em relação a temática, juntamente com suas percepções, e como tratamento para os dados foi utilizado o levantamento de categorias e a leitura reflexiva, para identificar as emergências centrais do tema (MARCELINO; PATRICIO, 20II).

O caráter metodológico de pesquisa exploratório também foi utilizado em uma das publicações, que coletou os dados com um protocolo de eliciamento de verbalização da conversa interna, a fim de captar e estudar o fenômeno a partir de uma estimulação prévia e empregando a fenomenologia semiótica para analisar e discutir os resultados da pesquisa (EHRENBRINK; SOUZA, 2018).

\section{METASSÍNTESE DOS DADOS QUALITATIVOS}

O estigma em torno da obesidade, discutido nessa pesquisa, foi visualizado nos quatro trabalhos. Decorente de comorbidades e limitações, têm-se o ônus emocional em relação a aparência do corpo estético pela hostilidade social face a obesidade (MARCELINO; PATRICIO, 20II). Este fator também se estente ao fato de que o temor da não aceitação do par, consoante as dimensões do corpo, faz com que o sujeito obeso crie uma coletânea de restrições afetivas e sexuais, engendradas pelo baixo sentimento de estima e valor (MARCELINO; PATRICIO, 2oII; MARCHESINI; ANTUNES, 2017).

O corpo obeso passa a ser estabelecido enquanto uma ameça a vida pela incubência da aversão social a gordura, fomentando os processos de exclusão intermediados e 
transmitidos em sua cotidianeidade (BENTO; MÉLO, 2org; EHRENBRINK; SOUZA, 2018). Como algo impresso no corpo, a obesidade leva o sujeito a uma esfera psíquica negativa, e daí possibilita a atenuação de sofrimentos psicológicos pelas narrativas psicossociais valorativas que entende o corpo gordo como feio e inaceitável, logo estigmatizado (MARCELINO; PATRICIO, 20II; MARCHESINI; ANTUNES, 20I7; BENTO; MÉLO, 2019).

Sendo assim, o processo da corporeidade é fundamentado pelas interações que ocorrem na conjuntura social, tornando o corpo como um instrumento de socialização (EHRENBRINK; SOUZA, 2018). Quando o corpo cede as pressões dessa socialização, busca métodos para resignificar o que foi vivido em seu corpo e projetar a sua nova modelação estética e todas as experiências futuras que lhe possibilitará, sendo a decisão da cirurgia bariátrica se torna um meio de escrever uma nova história a esse corpo, segundo os apontamentos (BENTO; MÉLO, 2019).

O engedramento dinâmico em torno da aprovação e desaprovação pelo grupo social, construiu no indivíduo pós-bariátrico um temor em relação a voltar a sua aparência antiga, no manejo que oreganho de peso é um alerta para o retorno à exclusão social, manifestado por discursos que incitam a ridicularização do corpo obeso, todavia, em contrapartida, o corpo mais magro, agora também mais ágil, possibilitou uma melhora no estado clínico geral e nas interações sociais, resgatando valores, autoestima e maior nível de confiança, isto é, reorientando a vida (MARCELINO; PATRICIO, 2oIr; MARCHESINI; ANTUNES, 2017; BENTO; MÉLO, 2019).

Por fim, pode ser visualizado que o indivíduo obeso se esforça para se enquadrar na sociedade que o discrimina, já que a sua essência é reduzida a sua aparência corporal e a sua distinção emr elação a um modelo o torna indigno de ser um ser social, havendo a necessidade de profissionais da psicologia estar atentos as peculiaridades do funcionamento dos processos reflexivos que levam os indivíduos obsesos a se submeterem para a cirurgia bariátrica em nome de um pertencimento estético instituído por representações imagéticas de uma beleza inalcançável.

\section{DISCUSSÕES}

A atuação profissional da psicologia no processo de cirurgia bariátrica, é entendida por Flores (2014) como muito valiosa, pois o psicólogo deve estar apto a avaliação das 
particularidades emocionais prévias acerca da história de vida pregressa, como também, do seu envolvimento com a cirurgia. A compreensão sobre o funcionamento psicológico do sujeito deve se fazer presente, para saber se este satisfaz critérios para a realização da bariátrica (FLORES, 2014).

Dentro da dinâmica psicossocial que o sujeito se encontra, é importante levantar informações a respeito da capacidade de boa aderência para mudanças, o comportamento alimentar, presença de comorbidades psiquiátricas, ideação suicida, uso substâncias psicoativas, funcionamento cognitivo, histórico de traumas e abusos, qualidade de vida e compreensão quanto ao risco da cirurgia (FLORES, 2014).

Amaral et. al. (202I), ressalta que a bariátrica se mostra extremamente eficaz em relação a perda de peso, redução de comorbidades e aumento da expectativa de vida, todavia, não pode deixar de lembrar que é uma cirurgia de alto risco, e por isso, envolve riscos de complicações pós-operatórios que vão desde obstrução intestinal até tromboembolismo e complicações cardiovasculares, dentre outras.

O alto risco que a cirurgia oferece deve ser levado em conta no momento de decisão de sua realização, posto que esta oportuniza certos bônus e ônus, sendo, desse modo, a prática da avaliação psicológica fundamental para não apenas identificar o preenchimento de critérios ou levantar as possíveis contraindicações, mas, primordialmente, corroborar um maior êxito no pós-cirúrgico e entender a motivação do indivíduo face esse processo de alto risco (FLORES, 2014).

Silva e Faro (2015), apontam que há uma pressão social para se adequar a estética vigente, e tal fato pode ser intermediado pela cirurgia bariátrica, pois a redução do estômago iria suscitar a adequação do corpo estético, e dessa maneira a realização do procedimento cirúrgico seria entendida e associada enquanto um ganho de imagem social, tornando-se um dos pontos nevrálgicos motivadores para a sua realização, e deixando a iminência dos riscos à saúde pelas complicações cirúrgicas em segundo plano

Nessa lógica, o processo cirúrgico passa a ser entendido e visualizado como uma solução rápida para a perda de peso, e consequentemente o ganho do corpo estético perfeito (SILVA; FARO, 2015). A ideia é de complacência diante dos mais diversos meios para resplandecer a imagem de um corpo estético condizente com os ditames estigmatizadores atuais (SILVA; FARO, 2015). 
Sendo assim, é possível visualizar a necessidade de entender a estética como motivador basilar para a realização da cirurgia bariátrica no processo de avaliação psicológica, face ao seu alto risco. Cabe ao profissional de psicologia o entendimento das percepções subjetivas, bem como as idealizações que o sujeito obeso apresenta em relação ao procedimento cirúrgico, visto que os ônus de sua decisão podem ser maiores do que os bônus da sua fantasia.

\section{CONSIDERAÇÕES FINAIS}

$\mathrm{Na}$ contemporaneidade, o corpo perdeu o seu valor de essência e passou a ser limitado a partir das medidas que o ideal estético impõem. A felicidade e o sucesso passaram a ser sinônimos de magreza, a aprovação é só para aqueles que são obedientes as regras, e a vergonha inscrita na própria imagem começou a imperar em quem ousa se opor a essa lógica perversa, deixando o indivíduo com uma mancha em sua imagem social.

A práxis e as discussões em torno da avaliação psicológica para bariátrica precisam avançar em torno das diversas variáveis que atravessam o corpo obeso, principalmente no que tange as reverberações do estigma e das práticas sociais de exclusão pela estética. É importante pensar em uma avaliação psicológica que abarque construtos válidos e pertinentes ao processo e as construções presentes nas configurações psicossociais do testando, ampliando as percepções em torno da complexidade relacional que situa o sujeito.

As considerações em torno do corpo estético precisam ser referendadas no processo de avaliação psicológica para bariátrica diante do entendimento de que esta se tornou um procedimento cirúrgico não para a saúde, mas para o corpo estético. O prisma de pensamento passa a ser de que o corpo obeso só será feliz, bem-sucedido e referido como um corpo belo se este for magro, ou seja, que reflete o ideal estético das narrativas sociais. Sendo assim, a real motivação em passar por uma cirurgia complexa, irreversível, de alto risco, e que pode desencadear complicações para a saúde física, emocional e psicológica deve ser investigado e discutido.

\section{REFERÊNCIAS BIBLIOGRÁFICAS}

AMARAL, Bruna Carolina Nogueira et al. Complicações decorrentes de cirurgia bariátrica em adultos jovens. Revista Eletrônica Acervo Científico, v. 33, p. e8047-e8047, 2021. Disponível em: 〈https://18.231.186.255/index.php/cientifico/article/view/8047〉. Acessado em o8 de Dezembro de 2021. 


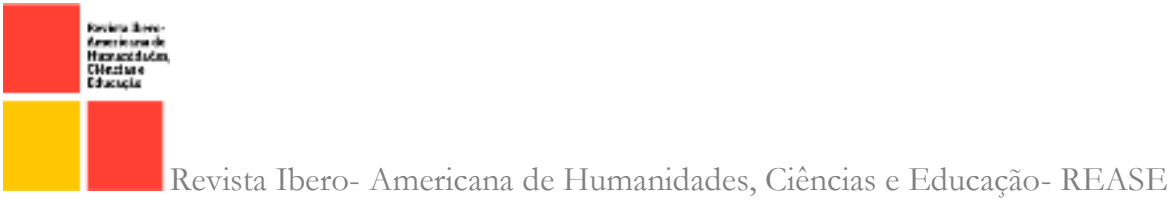

ARAÚJO, Kênya Lima de et al. Estigma do nutricionista com obesidade no mundo do trabalho. Revista de Nutrição [online]. 2015, v. 28, n. 6, pp. 569-579. Disponível em: 〈https://doi.org/ro.1590/I415-52732015000600ooI>. Acessado em 03 de dezembro de 2021.

BARBOSA, Bruno Rafael Silva Nogueira; DA SILVA, Laionel Vieira. A mídia como instrumento modelador de corpos: Um estudo sobre gênero, padrões de beleza e hábitos alimentares. Razón y Palabra, v. 20, n. 94, p. 672-687, set.-dez. 2016. Disponível em: 〈https://www.redalyc.org/pdf/r995/199547464041.pdf〉. Acessado em or de dezembro de 2021.

BENTO, Nárgila Mara da Silva; MÉLO, Roberta de Sousa. "A cabeça continua de gordo": Dilemas da gestão de si de pessoas submetidas a cirurgia bariátrica. Movimento (ESEFID/UFRGS), v. 25, p. 25073, 2019. Disponível em: 〈https://www.seer.ufrgs.br/Movimento/article/view/90445>. Acessado em o3 de dezembro de 202I.

COSTA, Anna Christina Charbel et al. Obesidade em pacientes candidatos a cirurgia bariátrica. Acta Paulista de enfermagem, v. 22, p. 55-59, 2009. Disponível me: 〈https://www.scielo.br/j/ape/a/gmtddzD6dDhFWn6FBhQnL6D/abstract/?lang=pt\&for mat $=h t m l>$. Acessado em o2 de dezembro de 202I.

DANTAS, Jurema Barros. Um ensaio sobre o culto ao corpo na contemporaneidade. 2011. Disponível em: 〈http://repositorio.ufc.br/handle/riufc/52775〉. Acessado em 30 de novembro de 2021.

DE VILHENA, Junia; MEDEIROS, Sergio; DE VILHENA NOVAES, Joana. A violência da imagem: estética, feminino e contemporaneidade. Revista Mal-estar e subjetividade, n. I, p. I09-I44, 2005. Disponível em: $\langle$ https://www.redalyc.org/pdf/27I/27150106.pdf $\rangle$. Acessado em 27 de novembro de 2021.

EHRENBRINK, Petra Paim; SOUZA, Mariane Lima de. Autoconsciência e conversa interna de adultos obesos à espera de cirurgia bariátrica: Um estudo qualitativo. Interação em psicologia, v. 22, n. I, 2018. Disponível em: $\langle$ https://revistas.ufpr.br/psicologia/article/view/53007〉. Acessado em 04 de dezembro de 2021.

FIGUEIREDO, Débora de Carvalho; NASCIMENTO, Fábio Santiago; RODRIGUES, Maria Eduarda. Discurso, culto ao corpo e identidade: representações do corpo feminino em revistas brasileiras. Linguagem em (Dis) curso, v. 17, n. I, p. 67-88, 2017. Disponível em: 〈https://www.scielo.br/scielo.php?pid=S1518-76322017000100067\&script=sci_arttext $>$. Acessado em 02 de dezembro de 2021.

FIORENTINI, Dario. Investigação em Educação Matemática desde a perspectiva acadêmica e profissional: desafios e possibilidades de aproximação. In: ANAIS do XIII Conferência Interamericana de Educação Matemática, CIAEM, 2014. Disponível em: 〈https://revistas.ucr.ac.cr/index.php/cifem/article/view/I47II〉. Acessado em or de dezembro de 202I. 
FLORES, Carolina Aita. Avaliação psicológica para cirurgia bariátrica: práticas atuais. Abcd. Arquivos Brasileiros de Cirurgia Digestiva (São Paulo), v. 27, p. 59-62, 2014. Disponível

em: <https://www.scielo.br/j/abcd/a/mpSh4vwswsHYhoLp3rVj6rC/?lang=pt\&format=html >. Acessado em 27 de novembro de 202I

GIL, A., C. Como Elaborar Projetos de Pesquisa. 4. ed. - São Paulo: Atlas, 2002.

GOMES, Isabelle Sena; DE OLIVEIRA CAMINHA, Iraquitan. Guia para estudos de revisão sistemática: uma opção metodológica para as Ciências do Movimento Humano. Movimento (ESEFID/UFRGS), v. 20, n. I, p. 395-411, 2014. Disponível em: <https://mail.google.com/mail/u/o/\#sent/WhctKJVRHCKRVnhtRgvbZHbXTqBjmvF ZjDfSNtMLZvKlllLwmkdGpvNrlscdpFnV1MQxbgL?projector=I\&amp; messagePartId=o .I\&gt>. Acessado em 02 de dezembro de 2021.

MACEDO, Tassia Teles Santana de et al. Percepção de pessoas obesas sobre seu corpo. Escola Anna Nery, v. 19, p. 505-510, 2015. Disponível em: 〈https://www.scielo.br/j/ean/a/xNzyMF7PDsyX8JkcxKxK8Bd/abstract/?lang=pt〉.

Acessado em 30 de novembro de 202I.

MARCELINO, Liete Francisco; PATRÍCIO, Zuleica Maria. A complexidade da obesidade e o processo de viver após a cirurgia bariátrica: uma questão de saúde coletiva. Ciência \& Saúde Coletiva, v. I6, p. 4767-4776, 20Ir. Disponível em: 〈https://www.scielo.br/j/csc/a/6C 3 YyYwLDLgrh6SdC5jDqBn/abstract/?lang=pt〉.

Acessado em 03 de dezembro de 2021.

MARCHESINI, Simone Dallegrave. Acompanhamento psicológico tardio em pacientes submetidos à cirurgia bariátrica. ABCD. Arquivos Brasileiros de Cirurgia Digestiva (São Paulo), v. 23, n. 2, p. 108-I13, 2010. Disponível em: $<$ https://www.scielo.br/scielo.php?pid=Soro2-

$67202010000200010 \&$ script $=$ sci_abstract $\& \ln \ln =e s>$. Acessado em 28 de novembro de 202I.

MARCHESINI, Simone Dallegrave; ANTUNES, Maria Cristina. A percepção do corpo em pacientes bariátricos e a experiência do medo do reganho do peso. Interação em Psicologia, v. 2I, n. 2, 2017. Disponível em: $\langle$ https://revistas.ufpr.br/psicologia/article/view/47944〉. Acessado em o4 de dezembro de 2021.

MATTOS, Rafael da Silva; LUZ, Madel Therezinha. Sobrevivendo ao estigma da gordura: um estudo socioantropológico sobre obesidade. Physis: Revista de Saúde Coletiva, v. $19, \quad$ p. $489-507, \quad 2009$. Disponível 〈https://www.scielo.br/j/physis/a/vGqBqHFkLxQ6x6Ydw5bK7Qq/abstract/?lang=pt〉. Acessado em 02 de dezembro de 2021.

OLIVEIRA, Verenice Martins de; LINARDI, Rosa Cardelino; AZEVEDO, Alexandre Pinto de. Cirurgia bariátrica: aspectos psicológicos e psiquiátricos. Archives of Clinical Psychiatry (São Paulo), v. 3I, n. 4, p. I99-20I, 2004. Disponível em: 
$<$ https://www.scielo.br/scielo.php?pid=SoroI-

$60832004000400014 \& s c r i p t=$ sci_arttext\&tlng=pt $>$. Acessado em 28 de novembro de 2021.

PAIM, Marina Bastos; KOVALESKI, Douglas Francisco. Análise das diretrizes brasileiras de obesidade: patologização do corpo gordo, abordagem focada na perda de peso e gordofobia. Saúde e Sociedade, v. 29, p. e190227, 2020. Disponível em: 〈https://www.scielosp.org/article/sausoc/2020.v29nI/er90227/pt/>. Acessado em $27 \mathrm{de}$ novembro de 202I.

RIBAS, Raíra Emanuelle Barbosa; CALEIRO, Maurício de Medeiros. Padrões estéticos e globalização: a sociedade pós-moderna frente à ditadura da beleza. Intercom-Sociedade Brasileira de Estudos Interdisciplinares da Comunicação, 2012. Disponível em: < http://www.intercom.org.br/papers/regionais/sudeste2012/resumos/r33-1567-I.pdf>.

Acessado em 27 de novembro de 202I.

SILVA, Cíntia; FARO, André. Significações relacionadas à cirurgia bariátrica: Estudo no pré e pós-operatório. Salud \& Sociedad, v. 6, n. 2, p. 156-169, 2015. Disponível em: $\langle$ https://dialnet.unirioja.es/servlet/articulo?codigo=6755298 $>$.Acessado em o9 de dezembro de 202I.

TAVARES, Maria da Conceição. Imagem Corporal: conceito e desenvolvimento. Barueri, SP: Manole, 2003. 